Novel diversely substituted 1-heteroaryl-2-imidazolines for fragment-based drug discovery

Mikhail Krasavin
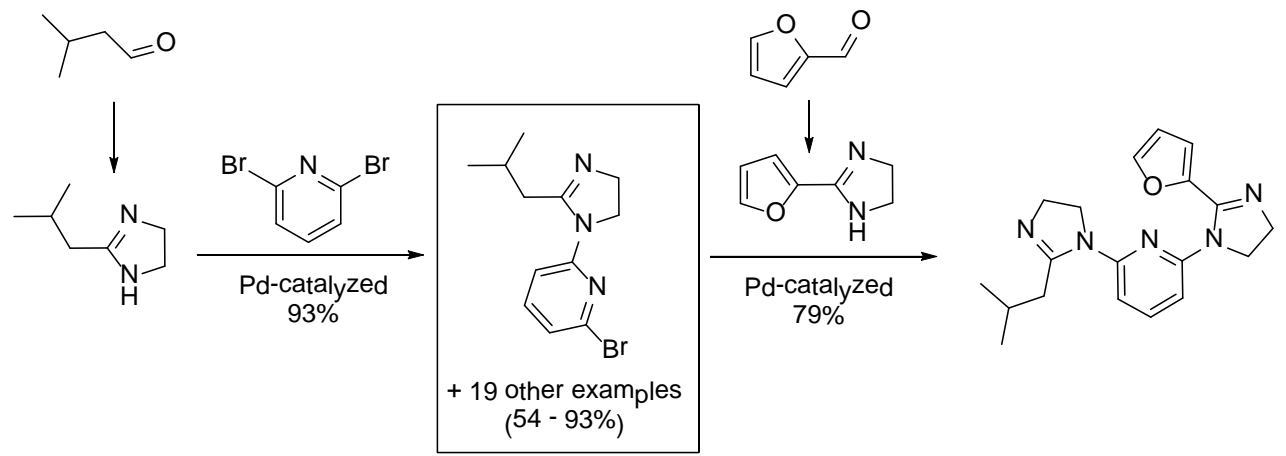


\title{
Novel diversely substituted 1-heteroaryl-2-imidazolines for fragment-based drug discovery
}

\author{
Mikhail Krasavin
}

Eskitis Institute for Cell and Molecular Therapies, Griffith University, Nathan, Queensland 4111, Australia

E-mail: ‥krasavin@griffith.edu.au, phone: +61 73735 6041, fax: +61 737356001

\begin{abstract}
A palladium-catalyzed Buchwald-Hartwig arylation protocol has been applied to achieve high-yielding $N$-heteroarylation of a diverse set of privileged 2-imidazolines. The resulting compounds are of interest as a novel type of molecular tool for fragment-based drug discovery. The potential for combining two 2-imidazoline moieties in a heteroarene-linked dimer via sequential Pd-catalyzed arylation has been demonstrated.
\end{abstract}

Keywords: fragment-based drug discovery, 2-imidazolines, Rule of Three, BuchwaldHartwig reaction, 2-haloazines, imidazoline dimers.

Since its invention at Abbott Laboratories in 1996, ${ }^{1}$ fragment-based drug discovery ${ }^{2}$ continues to evolve into a remarkably efficient alternative to the traditional high-throughput screening (HTS) technology of lead generation both in terms of compound numbers involved and the existing rational approaches for subsequent follow-up and optimization of the initial hits. ${ }^{3}$ In particular, X-ray crystallography guided optimization of fragments allows one to achieve significant increase in ligand affinity in strikingly few iterations. Success stories of such streamlined fragment-based drug discovery include nanomolar inhibitors of Aurora kinase $^{4}$ as well as heat shock protein 90 (Hsp90), ${ }^{5}$ which have advanced into clinical trials for cancer.

Typically, fragments display weaker ( $\mu \mathrm{M}$ to $\mathrm{mM}$ range) affinity to a biological target compared to traditional HTS hits. However, given their much smaller molecular weight, the ligand efficiency of fragment hits (i.e. binding energy per atom) ${ }^{6}$ is quite high. In designing new fragments, one should take into account certain guidelines restricting molecular weight ( $<300 \mathrm{Da}$ ) as well as other calculated properties of the future fragments (the so-called 'Rule of Three'). ${ }^{7}$ Moreover, fragment compounds should be sufficiently soluble to permit their

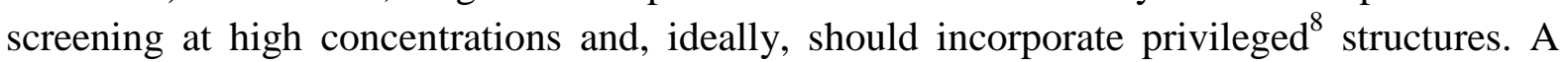
large number of privileged motifs have been identified to-date ${ }^{9}$ and are suggested as a basis for fragment design. ${ }^{10}$ However, many of these cores (in particular, fused aromatic coumarin, 
benzothiophene, quinolone, quinoxaline, etc.) are typically not conducive to high solubility of the resulting fragments.

In our program aimed at designing novel fragments for protein affinity screening, attention was devoted to the 2-imidazoline scaffold which has high solubility ${ }^{11}$ and can be regarded as being privileged. The latter aspect is clearly evidenced by the plethora of biological activities reported for 2-imidazoline-based compounds, such as binding to nicotinic acetylcholine receptors, ${ }^{12}$ inhibition of $\mathrm{K}^{+}$channels, ${ }^{13}$ suppression of NF-kB-mediated inflammatory response, ${ }^{14}$ antagonistic modulation of angiotensin II receptors, ${ }^{15}$ prominent adrenergic activity, ${ }^{16}$ and affinity to specialized imidazoline binding sites (or putative receptors) where discovery of certain subtype $\left(\mathrm{I}_{1}, \mathrm{I}_{2}, \mathrm{I}_{3}\right)$ selective modulators can lead to many useful therapeutic applications. ${ }^{17}$

2-Substituted 2-imidazolines $\mathbf{1}$ are very common and can be accessed in a variety of ways from readily available precursors, as recently reviewed by Crouch. ${ }^{18}$ We designed a series of 1,2-disubstututed imidazoline fragments 2 where an additional, preferably heteroaromatic moiety would contribute to the protein affinity, while the molecular weight of the resultant compounds would remain low $(<300 \mathrm{Da})$. Electron-deficient nitrogen heterocycles are expected to reduce the basicity (or cationic character ${ }^{19}$ ) of the imidazoline core (thereby reducing chances for non-specific protein binding) and provide opportunities for additional interactions with a protein (Figure 1). Retrosynthetically, $\mathbf{2}$ can be derived from $\mathbf{1}$ via heteroarylation of the latter with suitable heteroaromatic halides. Direct nucleophilic substitution of 2-chloroazines with 2-imidazolines has been reported to require the presence of an additional electron-withdrawing (such as nitro ${ }^{20}$ or carboxamide ${ }^{21}$ ) substituent in the azine coupling partner, thereby limiting the reaction scope. Transition metal catalyzed $\mathrm{N}$ arylation of amidines (2-imidazolines being a special case of a cyclic amidine) has been studied quite extensively, as recently reviewed by Maes et al. ${ }^{22}$ However, such methodology has not been developed for 2-imidazolines. ${ }^{23}$ Herein, we report on the preparation of diversely substituted, fragment-like $N$-heteroaryl 2-imidazolines via Pd-catalyzed BuchwaldHartwig arylation $^{24}$ of 2-substituted 2-imidazolines.

A series of 2-substituted 2-imidazolines 1a-k was synthesized on 10-40 mmol scale from the respective aldehyde precursors via condensation with ethylenediamine (EDA) and treatment with NBS (Scheme 1). ${ }^{25}$ Compounds $\mathbf{1 a - k}$ were isolated by simple crystallization or chromatographically in good to excellent yields (Table 1).

Having created a range of the starting materials for the target $N$-heteroaryl fragments 2 , we proceeded to identify a suitable $N$-arylation protocol. From the literature review ${ }^{22}$ on $N$ arylation of amidines, it appeared that methods employing copper-amine complexes to catalyse this reaction are as commonplace as those where palladium(II) complex catalysts are used. However, our attempts to $N$-arylate 1a with 2-chloropyrazine under such conditions as $\mathrm{CuI} / \mathrm{DMEDA} / \mathrm{K}_{2} \mathrm{CO}_{3} / \mathrm{DMF}, 8{ }^{\circ} \mathrm{C}, 12 \mathrm{~h}^{26}$ or CuI/L-proline/Cs $\mathrm{CO}_{3} / \mathrm{DMF}, 110{ }^{\circ} \mathrm{C}, 20 \mathrm{~h}^{27}$ led to negligible conversions and the formation of multiple by-products. In selecting an alternative, Pd-catalyzed protocol for the desired $N$-arylation, we were motivated by our prior experience in achieving $N$-arylation of poorly reactive heterocyclic amidines. ${ }^{28}$ The same 
protocol $^{29}$ was applied to imidazolines $1 \mathbf{a}-\mathbf{k}$ and a range of 2-haloazines to prepare a diverse set of the target fragment-like 1,2-disubstituted imidazolines 2a-t (Scheme 2). The isolated yields after column chromatography were generally good (Table 2). ${ }^{30}$ Notably, the molecular weights of compounds 2a-t did not exceed 300 Da thereby making these compounds excellent novel tools for fragment-based drug discovery. ${ }^{31}$

The scope of the Pd-catalyzed ${ }^{32} N$-(hetero)arylation protocol appears to be limited to highly reactive 2-haloazines. When applied to less reactive 5-bromopyrimidine or 4-cyano-2fluorophenyl bromide (which we considered sufficiently electron-deficient to participate in the Buchwald-Hartwig coupling, based on literature precedence ${ }^{33,34}$ ), the yields of the respective $N$-arylation products $\mathbf{2 u - v}$ were markedly lower (Scheme 3). Simple non-activated aryl halides (iodobenzene, 4-iodobiphenyl) or even 2-haloazines with electron-donating substituents (2-chloro-5-hydroxypyridine, 4-amino-2-chloropyrimidine) failed to undergo the desired reaction.

Finally, we were interested to determine if $N$-heteroaryl imidazolines containing reactive halogen substituents (such as 2c) could serve as a starting point for introducing further diversity to the novel $N$-heteroaryl 2-imidazoline scaffold. Quite satisfyingly, 2c could indeed be used to $N$-heteroarylate reactive secondary amines (such as morpholine or hexamethyleneimine) providing high yields of the fragment-like products 3a,b. Moreover, under the same conditions, $\mathbf{2 c}$ underwent a facile and high-yielding reaction with a second 2imidazoline providing access to a novel type of heteroarene-linked, non-symmetrical 2imidazoline dimer, 4 (Scheme 4). Dimeric imidazoline derivatives are of significant interest as potential anti-infective agents. ${ }^{35}$

In conclusion, we have identified a reliable protocol for $\mathrm{N}$-heteroarylation of privileged 2imidazolines and applied it to the synthesis of a diverse set of novel fragment-like compounds. ${ }^{36}$ These compounds will be screened for their affinity to therapeutically relevant proteins using Fourier-transform mass spectrometry ${ }^{37}$ and the results will be reported in due course.

\section{Acknowledgements}

The author acknowledges support from Griffith University (New Researcher Grant 2012, project 215586). Dr. Hoan Vu of Eskitis Institute is thanked for high-resolution mass spectrometry measurements.

\section{References and notes}

1. Shuker, S. B.; Hajduk, P. J.; Meadows, R. P.; Fesik, S. W. Science 1996, 274, 15311534.

2. Congreve, M.; Chessari, G.; Tisi, D.; Woodhead, A. J. J. Med. Chem. 2008, 51, 36613680 . 
3. Murray, C. W.; Rees, D. C. Nature Chem. 2009, 1, 187-192.

4. Howard, S.; Berdini, V.; Boulstridge, J. A.; Carr, M. G.; Cross, D. M.; Curry, J.; Devine, L. A.; Early, T. R.; Fazal, L.; Gill, A. L.; Heathcote, M.; Maman, S.; Matthews, J. E.; McMenamin, R. L.; Navarro, E. F.; O'Brien, M. A.; O'Reilly, M.; Rees, D. C.; Reule, M.; Tisi, D.; Williams, G.; Vinkovic, M.; Wyatt, P. G. J. Med. Chem. 2009, 52, 379-388.

5. Woodhead, A. J.; Angove, H.; Carr, M. G.; Chessari, G.; Congreve, M.; Coyle, J. E.; Cosme, J.; Graham, B.; Day, P. J.; Downham, R.; Fazal, L.; Feltell, R.; Figueroa, E.; Frederickson, M.; Lewis, J.; McMenamin, R.; Murray, C. W.; O'Brien, M. A.; Parra, L.; Patel, S.; Phillips, T.; Rees, D. C.; Rich, S.; Smith, D.-M.; Trewartha, G.; Vinkovic, M.; Williams, B.; Woolford, A. J.-A. J. Med. Chem. 2010, 53, 5956-5969.

6. Abadzapatero, C.; Metz, J. Drug Discovery Today 2005, 10, 464-469.

7. Congreve, M.; Carr, R.; Murray, C.; Jhoti, H. Drug Discovery Today 2003, 8, 876-877.

8. Evans, B. E. J. Med. Chem. 1988, 31, 2235-2246.

9. Welsch, M. E.; Shyder, S. A.; Stockwell, B. R. Curr. Opin. Chem. Biol. 2010, 14, 347361.

10. Barelier, S.; Krimm, I. Curr. Opin. Chem. Biol. 2011, 15, 469-474.

11. Remko, M.; Swart, M.; Bickelhaupt, F. M. Bioorg. Med. Chem. 2006, 14, 1715-1728.

12. Ferretti, G.; Dukat, M.; Giannella, M.; Piergentili, A.; Pigini, M.; Quaglia, W.; Damaj, M. I.; Matrin, B. R.; Glennon, R. A. J. Med. Chem. 2002, 45, 4724-4731.

13. Favaloro, J. L.; Andrews, K. L.; McPherson, G. A. Arch. Pharmacol. 2003, 367, 397405.

14. Kahlon, D. K.; Lansdell, T. A.; Fisk, J. S.; Hupp, C. D.; Friebe, T. L.; Hovde, S.; Jones, A. D.; Dyer, R. D.; Henry, R. W.; Tepe, J. J. J. Med. Chem. 2009, 52, 1302-1309.

15. Guo, X.-Z.; Shi, L.; Wang, R.; Liu, X.-X.; Li, B.-G.; Lu, X.-X. Bioorg. Med. Chem. 2008, 16, 10301-10310.

16. Hong, S.-S.; Bavadekar, S. A.; Lee, S.-I.; Patil, P. N.; Lalchandani, S. G.; Feller, D.R.; Miller, D. D. Bioorg. Med. Chem. Lett. 2005, 15, 4691-4695.

17. Li, J.-X.; Zhang, Y. Eur. J. Pharmacol. 2011, 658, 49-56 and references cited therein.

18. Crouch, R. D. Tetrahedron 2009, 65, 2387-2397.

19. Choi, Y.W.; Rogers, J. A. Pharm. Res. 1990, 7, 508-512.

20. Ryu, J. M.; Lee, J. S.; Park, W. J.; Yun, H.; Kim, K. Y. PCT Int. Appl. WO 2010007987, 175 pp.; Chem. Abstr. 2010, 153, 62137.

21. Caroff, E.; Fretz, H.; Hilpert, K.; Houille, O.; Hubler, F.; Meyer, E. PCT Int. Appl. WO 2006114774, 381 pp.; Chem. Abstr. 2006, 145, 471852.

22. Rauws, T. R. M.; Maes, B. U. W. Chem. Soc. Rev. 2012, 41, 2463-2497.

23. Several isolated examples of transition metal catalyzed arylation of azine scaffolds are available in the recent patent literature: (a) Galemmo, R. A., Jr.; Artis, D. R.; Ye, X. M.; Aubele, D. L.; Truong, A. P.; Bowers, S.; Hom, R. K.; Zhu, Y.-L.; Neitz, R. J.; Sealy, J.; Adler, M.; Beroza, P.; Anderson, J. P. PCT Int. Appl. WO 2011079118, 439 pp.; Chem. Abstr. 2011, 155, 152558; (b) Bamberg, J. T.; Bartlett, M.; Dubois, D. J.; Elworthy, T. R.; Hendricks, R. T.; Hermann, J. C.; Kondru, R. K.; Lemoine, R.; Lou, Y.; Owens, T. D.; Park, J.; Smith, D. B.; Soth, M.; Yang, H.; Yee, C. W. US Patent Appl. US 20090215750, 102 pp.; Chem. Abstr. 2009, 151, 313587. 
24. Buchwald, S. L.; Mauger, C.; Mignani, G.; Scholz, U. Adv. Synth. Catal. 2006, 348, 2339.

25. Fujioka, H.; Murai, K.; Ohba, Y.; Hiramatsu, A.; Kita Y. Tetrahedron Lett. 2005, 46, 2197-2199.

26. Yang, X.; Liu, H.; Fu, H.; Qiao, R.; Jiang, Y.; Zhao, Y. Synlett 2010, 101-106.

27. Huang, C.; Fu, Y.; Fu, H.; Jiang, Y.; Zhao, Y. Chem. Commun. 2008, 6333-6335.

28. Sandulenko, Y.; Komarov, A.; Rufanov, K.; Krasavin, M. Tetrahedron Lett. 2008, 49, 5990-5993.

29. General procedure $(0.5-3.0 \mathrm{mmol}$ scale $)$ for the preparation of compounds 2a-t: A thick-glass, screw-capped pressure tube $(50 \mathrm{~mL})$ was charged with a suspension of the starting imidazoline (1.0 equiv.), heteroaryl halide (1.1 equiv.) and $\mathrm{Cs}_{2} \mathrm{CO}_{3}$ (1.0 - 3.0 equiv., an additional equiv. per salt component of the reactants) in toluene ( $3 \mathrm{~mL} / \mathrm{mmol})$. $\mathrm{Pd}(\mathrm{OAc})_{2}$ (0.02 equiv.) and BINAP (0.4 equiv.) were weighed out into a vial, suspended in toluene $(2 \mathrm{~mL} / \mathrm{mmol})$ and shaken in a $100{ }^{\circ} \mathrm{C}$ oil bath for $2 \mathrm{~min}$. The resulting clear, purple catalyst solution was added at once to the vigorously stirred reaction mixture. The tube was filled with argon, capped, and stirred at $100{ }^{\circ} \mathrm{C}$ for $16-20 \mathrm{~h}$. The mixture was cooled, partitioned between EtOAc and water. The organic layer was separated, dried over anhydrous $\mathrm{MgSO}_{4}$, filtered, and concentrated in vacuo. The resulting crude material was purified by column chromatography on silica gel using an appropriate gradient of acetone in hexane (or $\mathrm{MeOH}$ in $\mathrm{CH}_{2} \mathrm{Cl}_{2}$ ) as eluent to provide the target product 2 .

30. Characterization data for representative compounds: 2a - 85\% yield (30\% acetone in hexane), clear oil. ${ }^{1} \mathrm{H}$ NMR (500 MHz, $\left.\mathrm{CDCl}_{3}\right) \delta 8.52$ (d, $\left.J=5.0 \mathrm{~Hz}, 1 \mathrm{H}\right), 7.76-7.81(\mathrm{~m}$, 2H), 7.45 (m, 1H), 7.16-7.21 (m, 2H), $4.11(\mathrm{t}, J=8.5 \mathrm{~Hz}, 2 \mathrm{H}), 3.87$ (t, $J=8.5 \mathrm{~Hz}, 2 \mathrm{H})$, 3.00 (d, $J=7.0 \mathrm{~Hz}, 2 \mathrm{H}$ ), 2.16 (sept, $J=7.0 \mathrm{~Hz}, 1 \mathrm{H}$ ), 1.01 (d, $J=6.5 \mathrm{~Hz}, 6 \mathrm{H}$ ); ${ }^{13} \mathrm{C} \mathrm{NMR}$ $\left(125 \mathrm{MHz}, \mathrm{CDCl}_{3}\right) \delta 163.2$ (d, $\left.J_{C-F}=245.0 \mathrm{~Hz}\right), 163.1$ (d, $J_{C-F}=2.5 \mathrm{~Hz}$ ), 161.5, 158.5, 158.1, 139.4 (d, $\left.J_{C-F}=7.5 \mathrm{~Hz}\right), 122.6$ (d, $\left.J_{C-F}=2.5 \mathrm{~Hz}\right), 117.7$ (d, $J_{C-F}=21.3 \mathrm{~Hz}$ ), 114.0 $\left(\mathrm{d}, J_{C-F}=23.8 \mathrm{~Hz}\right), 108.7,51.6,48.7,40.8,26.1,22.6$; HRMS $\mathrm{m} / \mathrm{z}[\mathrm{M}+\mathrm{H}]^{+}$calcd for $\mathrm{C}_{17} \mathrm{H}_{20} \mathrm{FN}_{4}$ 299.1667, found 299.1654. 2h - 91\% yield (30\% acetone in hexane), waxy solid. ${ }^{1} \mathrm{H}$ NMR (500 MHz, $\mathrm{CDCl}_{3}$ ) $\delta 8.49$ (d, $J=4.8 \mathrm{~Hz}, 2 \mathrm{H}$ ), 7.94 (s, 2H), 7.60 (dd, $J=$ 7.7, $1.5 \mathrm{~Hz}, 1 \mathrm{H}), 7.28\left(\mathrm{td}, J_{t}=7.7 \mathrm{~Hz}, J_{d}=1.5 \mathrm{~Hz}, 1 \mathrm{H}\right), 6.96(\mathrm{t}, J=7.5 \mathrm{~Hz}, 1 \mathrm{H}), 6.89(\mathrm{~d}$, $J=8.2 \mathrm{~Hz}, 1 \mathrm{H}), 6.77(\mathrm{t}, J=4.8 \mathrm{~Hz}, 1 \mathrm{H}), 4.11(\mathrm{t}, J=9.1 \mathrm{~Hz}, 2 \mathrm{H}), 3.98(\mathrm{t}, J=9.1 \mathrm{~Hz}$, 2H), 3.86 (s, 3H); ${ }^{13} \mathrm{C}$ NMR (125 MHz, $\left.\mathrm{CDCl}_{3}\right) \delta 158.7,158.2,157.8,157.6,132.2$, 129.9, 127.9, 125.6, 120.5, 119.7, 113.2, 111.0, 55.4, 52.0, 48.8; HRMS m/z [M+H] ${ }^{+}$ calcd for $\mathrm{C}_{16} \mathrm{H}_{17} \mathrm{~N}_{4} \mathrm{O}$ 281.1397, found 281.1384. $2 \mathbf{p}-62 \%$ yield (40 $\rightarrow 50 \%$ acetone in hexane), white solid, mp $=123-125{ }^{\circ} \mathrm{C} .{ }^{1} \mathrm{H}$ NMR (500 MHz, $\left.\mathrm{CDCl}_{3}\right) \delta 8.06$ (d, $J=4.6$ $\mathrm{Hz}, 1 \mathrm{H}$ ), 7.36 (ddd, $J=11.6,8.1,1.4 \mathrm{~Hz}, 1 \mathrm{H}), 7.27$ (d, $J=1.0 \mathrm{~Hz}, 1 \mathrm{H}), 7.04$ (ddd, $J=$ 8.1, 4.6, 3.4 Hz, 1H), 6.63 (d, $J=3.4 \mathrm{~Hz}, 1 \mathrm{H}), 6.35$ (dd, $J=3.4,1.8 \mathrm{~Hz}, 1 \mathrm{H}), 4.08$ (m, 2H), $4.01(\mathrm{~m}, 2 \mathrm{H}) ;{ }^{13} \mathrm{C}$ NMR (125 MHz, $\left.\mathrm{CDCl}_{3}\right) \delta 153.1,151.7$ (d, $\left.J_{C-F}=257.5 \mathrm{~Hz}\right)$, 145.6, $144.4\left(\mathrm{~d}, J_{C-F}=11.3 \mathrm{~Hz}\right), 143.3\left(\mathrm{~d}, J_{C-F}=5.0 \mathrm{~Hz}\right), 143.2,123.9$ (d, $J_{C-F}=17.5$ $\mathrm{Hz}), 120.8$ (d, $\left.J_{C-F}=2.5 \mathrm{~Hz}\right), 112.4,111.1,54.2,51.6$ (d, $J_{C-F}=1.3 \mathrm{~Hz}$ ); HRMS m/z $[\mathrm{M}+\mathrm{Na}]^{+}$calcd for $\mathrm{C}_{12} \mathrm{H}_{10} \mathrm{FN}_{3} \mathrm{ONa}$ 254.0700, found 254.0692.

31. Although we did not perform quantitative solubility measurements for compounds 2a-t, all were remarkably soluble in polar organic solvents $\left(\mathrm{CH}_{2} \mathrm{Cl}_{2}, \mathrm{CHCl}_{3}, \mathrm{MeOH}, \mathrm{EtOAc}\right)$. 
In order to test for solubility in aqueous medium, we prepared a $100 \mathrm{mM}$ solution of a representative compound $2 \mathrm{t}$ in methanol and added $10 \mu \mathrm{L}$ of this solution to a $50 \mathrm{x}$ volume of $\mathrm{pH} 7.2$ phosphate buffer solution. There was no observable precipitation.

32. In a control experiment, reaction of $\mathbf{1 k}$ with 2-bromopyrimidine under the general procedure conditions, but in the absence of $\mathrm{Pd}(\mathrm{OAc})_{2}$, did not produce any of the arylation product $2 \mathbf{o}$.

33. Kim, G. S.; Cho, Y. J.; Kwon, H. J.; Kim, B. O.; Kim, S. M.; Yoon, S. S. Eur. Pat. Appl. EP 2096108, 356 pp.; Chem. Abstr. 2009, 151, 300937.

34. Wood, H. B.; Adams, A. D.; Freeman, S.; Szewczyk, J. W.; Santini, C.; Huang, Y. PCT Int. Appl. WO 2008085316, 152 pp.; Chem Abstr. 2008, 149, 176187.

35. Jacobs, M. R.; Bajaksouziana, S.; Good, C. E.; Butler, M. M.; Williams, J. D.; Peet, N. P.; Bowlin, T. L.; Endimiani, A.; Bonomo, R. A. Diagnostic Microbiol. Infect. Dis. 2011, 69, 114-116.

36. All of the compounds synthesized in this work have been deposited with the Queensland Compound Library (Griffith University) and are available for collaborative discovery projects.

37. Vu, H.; Pham, N. B.; Quinn, R. J. J. Biomol. Screening 2008, 13, 265-275. 
Scheme 1. Preparation of 2-substituted 2-imidazolines.

$$
\mathrm{R} \text {-1 } \frac{\text { 1) EDA }(1.05 \text { equiv. }), \mathrm{CH}_{2} \mathrm{Cl}_{2}, 0{ }^{\circ} \mathrm{C}, 20 \mathrm{~min}}{\text { 2) } \mathrm{NBS}(1.05 \text { equiv. }), \mathrm{CH}_{2} \mathrm{Cl}_{2}, 0^{\circ} \mathrm{C}->\text { r. t., } 16 \mathrm{~h}}
$$

Scheme 2. $N$-heteroarylation of 2-imidazolines 1a-k.<smiles>[R]C1=NCCN1</smiles>

1a-k

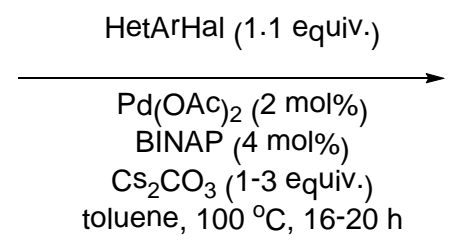

toluene, $100{ }^{\circ} \mathrm{C}, 16-20 \mathrm{~h}$

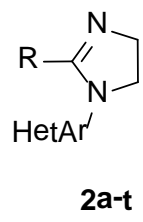

2a-t

Scheme 3. Examples of less reactive haloaromatics.<smiles>c1coc(C2=NCCN2)c1</smiles>

1b

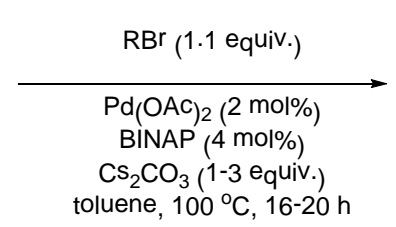

toluene, $100{ }^{\circ} \mathrm{C}, 16-20 \mathrm{~h}$<smiles>[R]N1CCN=C1c1ccco1</smiles><smiles></smiles>

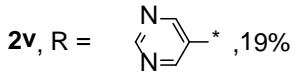

Scheme 4. Further Buchwald-Hartwig reactions of compound 2c.<smiles>CC(C)CC1=NCCN1c1cccc(N2CCN=C2c2ccco2)c1</smiles>

$4,79 \%$

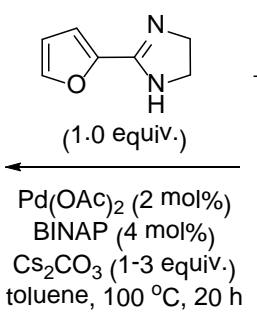

$\mathrm{Cs}_{2} \mathrm{CO}_{3}(1-3$ equiv. $)$
toluene, $100{ }^{\circ} \mathrm{C}, 20 \mathrm{~h}$

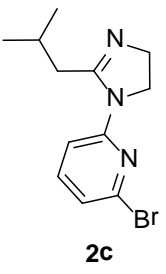

2c
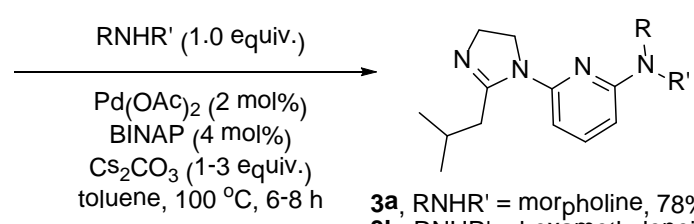

3a, $\mathrm{RNHR}^{\prime}=$ morpholine, $78 \%$

3b, RNHR' = hexamethyleneimine, $83 \%$

Figure 1. 2-Substituted 2-imidazolines (1) and the design of the new 1-heteroaryl 2imidazoline fragments (2).

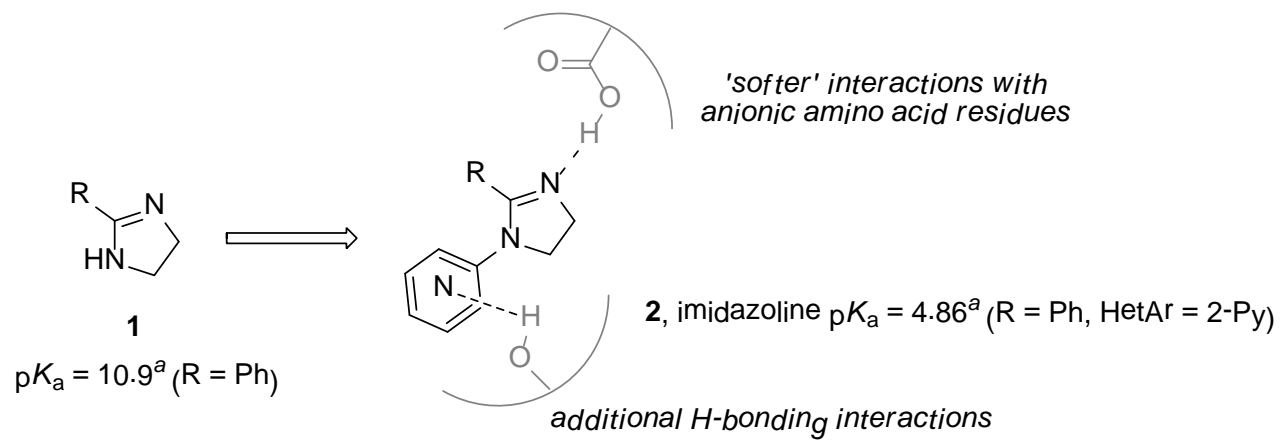

\footnotetext{
${ }^{a} \mathrm{p} K_{\mathrm{a}}$ value was calculated using ACD Labs 6.00 software.
} 
Table 1. 2-Substituted 2-imidazolines 1a-k prepared in this work.

\begin{tabular}{|c|c|c|}
\hline Compound & Structure & $\begin{array}{l}\text { Isolated } \\
\text { yield, \% }\end{array}$ \\
\hline & & \\
\hline & & $94^{b}$ \\
\hline & & $89^{a}$ \\
\hline & & $67^{C}$ \\
\hline & & $63^{b}$ \\
\hline 1e & & \\
\hline If & & $89^{b}$ \\
\hline & & $96^{b}$ \\
\hline & & $63^{b}$ \\
\hline in & & \\
\hline $1 \mathbf{i}$ & & \\
\hline & & $48^{b}$ \\
\hline & & $56^{C}$ \\
\hline $1 \mathrm{k}$ & & \\
\hline
\end{tabular}

${ }^{a}$ Isolated by conversion of the crude product into a hydrochloride salt and crystallization of the latter from $\mathrm{MeOH}$.

${ }^{b}$ Isolated by crystallization of the crude product from ethyl acetate-hexanes.

${ }^{c}$ Isolated by column chromatography on silica gel using $\mathrm{CH}_{2} \mathrm{Cl}_{2}-\mathrm{MeOH}-28 \%$ aq. $\mathrm{NH}_{4} \mathrm{OH}$ (90:9.5:0.5) as eluent. 
Table 2. $N$-heteroaryl 2-imidazolines 2a-t prepared in this work.

\begin{tabular}{|c|c|c|c|c|c|c|c|}
\hline Compound & $\begin{array}{l}\text { Starting } \\
\text { material }\end{array}$ & Halide & $\mathrm{R}$ & HetAr & $\begin{array}{l}\text { Isolated } \\
\text { yield, \% }\end{array}$ & MW & $\begin{array}{c}\mathrm{MS}[\mathrm{M}+\mathrm{H}]^{+} \\
\mathrm{m} / \mathrm{z}\end{array}$ \\
\hline $2 a$ & $1 \mathbf{a}$ & $\mathrm{Cl}$ & & & 85 & 298.4 & 299.3 \\
\hline $2 \mathbf{b}$ & $1 \mathbf{i}$ & $\mathrm{Br}$ & & & 78 & 242.3 & 243.3 \\
\hline $2 c$ & $1 \mathrm{a}$ & $\mathrm{Br}$ & & & 93 & 281.2 & 282.2 \\
\hline $2 d$ & 1d & $\mathrm{Cl}$ & & & 66 & 285.4 & 286.4 \\
\hline $2 e$ & $1 e$ & $\mathrm{Cl}$ & & & 68 & 263.3 & 264.3 \\
\hline $2 f$ & 1f & $\mathrm{Cl}$ & & & 54 & 295.3 & 296.2 \\
\hline $2 g$ & $1 \mathrm{~g}$ & $\mathrm{Cl}$ & & & 65 & 239.3 & 240.3 \\
\hline $2 h$ & 1c & $\mathrm{Br}$ & & & 91 & 280.3 & 281.2 \\
\hline $2 \mathbf{i}$ & $1 e$ & $\mathrm{Cl}$ & & & 92 & 264.3 & 265.4 \\
\hline $2 \mathbf{j}$ & $1 \mathbf{a}$ & $\mathrm{Cl}$ & & & 66 & 280.4 & 281.4 \\
\hline $2 \mathbf{k}$ & $1 \mathbf{a}$ & $\mathrm{Cl}$ & & & 78 & 218.3 & 219.3 \\
\hline 21 & $1 \mathbf{a}$ & $\mathrm{Cl}$ & & & 74 & 204.3 & 205.3 \\
\hline $2 m$ & $1 \mathrm{~h}$ & $\mathrm{Cl}$ & & & 81 & 239.3 & 240.4 \\
\hline $2 n$ & $1 \mathrm{~h}$ & $\mathrm{Cl}$ & & & 76 & 300.4 & 301.4 \\
\hline 20 & $1 k$ & $\mathrm{Br}$ & & & 61 & 268.4 & 269.4 \\
\hline $2 p$ & $1 b$ & $\mathrm{Cl}$ & & & 62 & 231.2 & 232.3 \\
\hline $2 q$ & 1c & $\mathrm{Cl}$ & & & 48 & 280.3 & 281.4 \\
\hline $2 r$ & $1 \mathrm{~g}$ & $\mathrm{Br}$ & & & 85 & 242.3 & 243.3 \\
\hline $2 s$ & $1 b$ & $\mathrm{Cl}$ & & & 63 & 290.3 & 291.3 \\
\hline $2 t$ & $\mathbf{1 j}$ & $\mathrm{Br}$ & & & 93 & 288.7 & 289.3 \\
\hline
\end{tabular}

